\title{
Chronic Periodontitis is Associated With Cerebral Atherosclerosis -A Nationwide Study
}

Urvish K. Patel ${ }^{1}$, Preeti Malik ${ }^{2,3}$, Nishanth Kodumuri ${ }^{4}$, Priyadarshee Patel ${ }^{5}$, Varun Pitti ${ }^{6}$, Gaurav Tyagi ${ }^{6}$ , Bindi Chauhan ${ }^{7}$, Abhishek Lunagariya ${ }^{8}$, Ravish Kothari ${ }^{4}$, Souvik Sen ${ }^{4}$

1. Neurology and Public Health, Icahn School of Medicine at Mount Sinai, New York, USA 2. Public Health, Icahn School of Medicine at Mount Sinai, New York, USA 3. Neurology, Massachusetts General Hospital, Andover, USA 4. Neurology, Palmetto Health-University of South Carolina School of Medicine, Columbia, USA 5. Neurology, Drexel University College of Medicine, Philadelphia, USA 6. Dentistry, Rutgers School of Dental Medicine, Newark, USA 7. Public Health, Long Island University, New York, USA 8. Neurology, Creighton University School of Medicine, Omaha, USA

Corresponding author: Urvish K. Patel, dr.urvish.patel@gmail.com

\section{Abstract \\ Introduction}

Chronic periodontitis and atherosclerosis share common risk factors and produce the same inflammatory markers. Many studies found a high prevalence of chronic periodontitis in patients with atherosclerosis but there is no strong evidence to support a specific association of chronic periodontitis with cerebral atherosclerosis. We aimed to study the concurrent prevalence and association of chronic periodontitis with cerebral atherosclerosis and cerebrovascular diseases among the US population.

\section{Methods}

We performed a cross-sectional analysis of a Nationwide Inpatient Sample with adult hospitalizations to identify the primary diagnosis of cerebrovascular diseases [acute ischemic stroke (AIS), hemorrhagic stroke (HS), and transient ischemic attack (TIA)] with concurrent cerebral atherosclerosis and chronic periodontitis. Multivariate survey logistic regression models were fitted to evaluate the linkage of chronic periodontitis with cerebral atherosclerosis and cerebrovascular diseases.

\section{Results}

Of total $56,499,788$ hospitalizations, $0.01 \%$ had chronic periodontitis. Prevalence of chronic periodontitis was higher in $50-64$ years ( $36.18 \%$ vs. $23.91 \%$ ), males ( $59.19 \%$ vs. $41.06 \%$ in females), Afro-Americans ( $25.93 \%$ vs. $15.21 \%$ ), and $0-25$ th percentile median-household-income-category (38.31\% vs. $30.15 \%$ ) compared to non-chronic periodontitis. There was significantly higher prevalence of cerebral atherosclerosis ( $0.71 \%$ vs. $0.41 \%$; $p<0.0001$ ) with weak evidence of high prevalence of cerebrovascular diseases (AIS:2.21\% vs. $1.97 \%$; $\mathrm{p}=0.1563$; HS:0.57\% vs. $0.46 \%$; $\mathrm{p}=0.1560$ ) among chronic periodontitis compared to non-chronic periodontitis. In regression analysis, odds of having cerebral atherosclerosis were 2.48 -folds higher in patients with chronic periodontitis compared to that without-chronic periodontitis, and cerebral atherosclerosis patients were associated with higher odds of TIA (aOR:2.40; $\mathrm{p}<0.0001)$, AIS (aOR:3.35; $\mathrm{p}<0.0001$ ), and $\mathrm{HS}$ (aOR:1.51; $\mathrm{p}<0.0001)$ compared to without-cerebral atherosclerosis. No significant relationship between chronic periodontitis and cerebrovascular diseases was observed.

\section{Conclusion}

Although chronic periodontitis may not directly increase the risk of cerebrovascular diseases, it increases the burden of cerebrovascular diseases by evidently increasing the risk of cerebral atherosclerosis. Early identification of chronic periodontitis and atherosclerotic risk factors may help to mitigate the risk of cerebrovascular diseases.

\section{Categories: Neurology, Public Health, Epidemiology/Public Health}

Keywords: cerebral atherosclerosis, chronic periodontitis, cerebrovascular disease, atherosclerosis, acute ischemic stroke, hemorrhagic stroke, transient ischemic attack, nationwide inpatient sample (nis), chronic inflammation, cardiovascular disease

\section{Introduction}

Chronic periodontitis is a chronic inflammation caused by bacterial colonization that affects the periodontal tissue supporting the teeth. The prevalence of chronic periodontitis is high, affecting $90 \%$ of the global population and therefore contributes significantly to the global burden of chronic diseases [1,2]. Hence, chronic periodontitis is arising as a major public health issue. According to a recent report from the Centers for Disease Control and Prevention (CDC), $47.2 \%$ of adults $>30$ years of age have periodontitis which increases with age and $70.1 \%$ of people by $>65$ years of age have chronic periodontitis [3]. 
Chronic Periodontitis can be a risk factor for many systemic diseases like cardiovascular disease, carotid artery disease, cerebrovascular disease, etc. contributing significantly to mortality and morbidity worldwide due to common inflammatory pathogenesis $[1,4,5]$. Studies have shown that chronic periodontitis patients are prone to the dissemination of oral bacteria and endotoxins into the bloodstream, which can trigger a pro-atherogenic response in endothelial cells leading to systemic inflammation and fibrin deposition. Eventually, this increased systemic inflammatory response is associated with the onset and progression of atherosclerosis and cerebrovascular disease [6-9]. Chronic periodontitis shares many concurrent conditions and risk factors for vascular disorders like atherosclerosis, stroke, cardiovascular disease, hypertension, dyslipidemia, diabetes mellitus, and smoking [10].

The exact prevalence of periodontitis in a patient with cerebral atherosclerosis and cerebrovascular diseases is not known. Few meta-analyses including both retrospective and prospective studies have reported a higher risk of cardio- and cerebrovascular disease in patients with chronic periodontitis [5,11-14]. But subtypes of stroke such as acute ischemic stroke (AIS), hemorrhagic stroke (HS), and transient ischemic attack (TIA) have not been analyzed separately in those meta-analyses. Also, those meta-analyses were from small cohort observational studies, and the estimated association may not be true considering the influence of biases (selection and observer) and confounding factors. All these limitations with previous studies prompt the need for a large population-based study to determine the prevalence of chronic periodontitis in a large group of patients with cerebral atherosclerosis and cerebrovascular diseases.

In this cross-sectional retrospective study, we aimed to describe the prevalence of cerebral atherosclerosis and cerebrovascular diseases among a large cohort of patients with concurrent chronic periodontitis and to assess whether chronic periodontitis is associated with cerebral atherosclerosis and cerebrovascular diseases like AIS, HS, and TIA.

\section{Materials And Methods}

We have obtained Nationwide Inpatient Sample (NIS) data from the Healthcare Cost and Utilization Project (HCUP) between January 2013 and December 2014. The NIS is the all-payer inpatient care database in the US and contains discharge-level data provided by states that participate in the HCUP. This dataset acquires approximately $20 \%$ of the stratified sample of all US community hospitals, representing more than $95 \%$ of the national population. Each hospitalization is treated as an individual entry in the database and is coded with one principal diagnosis and up to 24 secondary diagnoses associated with that stay. Detailed information on NIS is available at http://www.hcup-us.ahrq.gov/db/nation/nis/nisdde.jsp. The NIS is a deidentified database, so informed consent or IRB approval was not needed for the study. The HCUP Data Use Agreement (HCUP-348L73IZS) for the data utilized in this study was obtained. [15]

\section{Study population}

We used the 9th revision of the International Classification of Diseases, clinical modification codes (ICD-9$\mathrm{CM}$ ) to identify adult patients admitted with a primary and secondary diagnosis of chronic periodontitis (ICD-9-CM code 523.4) [11]. Similarly, patients with concurrent diagnosis of cerebral atherosclerosis (atheroma of cerebral arteries; ICD-9-CM code 437.0), transient ischemic attack (ICD-9-CM code 435), acute ischemic stroke (ICD-9-CM codes 433.01, 433.11, 433.21, 433.31, 433.81, 433.91, 434.01, 434.11, 434.91), and hemorrhagic stroke (ICD-9-CM codes 430, 431) were identified. The ischemic stroke and transient ischemic attack codes have 35\% sensitivity, 99\% specificity, 96\% PPV (positive predictive values), and 79\% NPV (negative predictive values); hemorrhagic stroke codes have $60 \%$ sensitivity, >99\% specificity, $77 \%$ PPV, and >99\% NPV; and cerebrovascular diseases combined codes have $46.3 \%$ sensitivity, $99.1 \%$ specificity, 81.2\% PPV, and 95.4\% NPV [16]. We used ICD-9-CM codes to identify the comorbidities of diabetes mellitus, hypertension, obesity, hypercholesterolemia, current or past smoker, drug abuse/dependent, alcohol abuse/dependent, AIDS, renal failure (Acute/ Chronic/ ESRD). Table 1 lists all ICD-9-CM codes that were used for this study. Age < 18 years and admissions with missing data for age, sex, and race were excluded. 


\section{Cureus}

Condition

HIVIAIDS

Hypercholesterolemia

Obesity

Hypertension

Diabetes Mellites

Alcohol abuse/dependent

Current or past smoker

Drug abuse

Renal failure (Acute/ Chronic/ ESRD)

\section{ICD-9 CM Codes}

042, V08

$272.0,272.1,272.2$

$278.00,278.01,278.02$

401-405

$249.00,250$

V11.3, 303, 305.0

V15.82, 305.1

$304,305.2-305.9$

$584,585,585.6$

\section{TABLE 1: ICD-9-CM codes used in this analysis}

\section{Patient and hospital characteristics}

Patient characteristics of interest were age, sex, race, insurance status, comorbidities, and concomitant diagnoses as defined above. The race was defined by white (referent), African American, Hispanic, Asian or Pacific Islander, and Native American. Insurance status was defined by Medicare (referent), Medicaid, Private Insurance, and Other/Self-pay/No charge. We defined the severity of co-morbid conditions using Deyo's modification of the Charlson co-morbidity index (CCI) (Table 2).

\begin{tabular}{|c|c|c|}
\hline Condition & ICD-9-CM Codes & Charlson Score \\
\hline Myocardial infarction & $410-410.9$ & 1 \\
\hline Congestive heart failure & $428-428.9$ & 1 \\
\hline Peripheral vascular disease & $433.9,441-441.9,785.4, \mathrm{~V} 43.4$ & 1 \\
\hline Cerebrovascular disease & $430-438$ & 1 \\
\hline Dementia & $290-290.9$ & 1 \\
\hline Chronic pulmonary disease & $490-496,500-505,506.4$ & 1 \\
\hline Rheumatologic disease & 710.0, 710.1, 710.4, 714.0 - 714.2, 714.81, 725 & 1 \\
\hline Peptic ulcer disease & $531-534.9$ & 1 \\
\hline Mild liver disease & $571.2,571.5,571.6,571.4-571.49$ & 1 \\
\hline Diabetes & $250-250.3,250.7$ & 1 \\
\hline Diabetes with chronic complications & $250.4-250.6$ & 2 \\
\hline Hemiplegia or paraplegia & $344.1,342-342.9$ & 2 \\
\hline Renal disease & $582-582.9,583-583.7,585,586,588-588.9$ & 2 \\
\hline Any malignancy including leukemia and lymphoma & 140-172.9, 174-195.8, 200-208.9 & 2 \\
\hline Moderate or severe liver disease & $572.2-572.8$ & 3 \\
\hline Metastatic solid tumor & 196-199.1 & 6 \\
\hline AIDS & 042 & 6 \\
\hline
\end{tabular}

TABLE 2: Deyo's modification of Charlson's co-morbidity index (CCI) 


\section{Outcomes}

The primary aim of the study is to identify the prevalence of cerebral atherosclerosis and cerebrovascular diseases (TIA, AIS, and HS) among patients with concurrent chronic periodontitis. The secondary aim is to evaluate the association of cerebral atherosclerosis and cerebrovascular diseases with chronic periodontitis.

\section{Statistical analysis}

All statistical analyses were performed using the weighted survey methods in SAS (version 9.4). Weighted values of patient-level observations were generated to produce a nationally representative estimate of the entire US population of hospitalized patients. P-values of $<0.05$ was considered significant. Univariate analysis of differences between categorical variables was tested using the chi-square test. The mixed-effects survey logistic regression models with weighted analysis were used for the dependent variables, in order to estimate odds ratio (OR) and 95\% Confidence Interval for the evaluation of the relationship of chronic periodontitis with cerebral atherosclerosis and cerebrovascular diseases and the relationship of cerebral atherosclerosis with cerebrovascular diseases.

In the multivariable analysis, we included demographics (age, gender, race), patient-level hospitalization variables (admission day, primary payer, admission type, Median Household Income Category), hospitallevel variables (hospital region, teaching versus nonteaching hospital, hospital bed size), comorbidities like diabetes mellitus, hypertension, obesity, hypercholesterolemia, current or past smoker, drug abuse/dependent, alcohol abuse/dependent, AIDS, renal failure (Acute/Chronic/End-stage renal disease or ESRD) and CCI.

For each model, c-index (a measure of goodness of fit for binary outcomes in a logistic regression model) was calculated. All statistical tests used were 2 -sided, and $\mathrm{p}<0.05$ was deemed statistically significant. No statistical power calculation was conducted prior to the study.

\section{Data availability}

The data that support the findings of this study are publicly available from the Agency for Healthcare Research and Quality's Healthcare Cost and Utilization Project (HCUP) Nationwide Inpatient Sample (NIS). A raw analysis of the data will be however made available from the authors upon request and with permission of HCUP-NIS.

\section{Results \\ Disease hospitalizations}

There was a total of 56,499,768 hospitalizations in the United States from year January 2013 to December 2014 after excluding patients with age $<18$ years and admissions with missing data for age, gender, and race. Out of 56,499,768 hospitalizations, 7020 (0.01\%) had chronic periodontitis.

\section{Demographics, patient and hospital characteristics, and comorbidities}

In the US hospitalizations, the prevalence of chronic periodontitis was higher in patients age group 50-64 years ( $36.18 \%$ vs. $23.91 \%$, $\mathrm{p}<0.0001)$, male ( $59.19 \%$ vs. $41.06 \%$, $\mathrm{p}<0.0001)$, African American ( $25.93 \%$ vs. $15.21 \%, \mathrm{p}<0.0001$ ), and $0-25$ th percentile median household income category ( $38.31 \%$ vs. $30.15 \%, \mathrm{p}<0.0001$ ) compared to non-chronic periodontitis. The frequency of chronic periodontitis was higher among patients with co-morbidities such as obesity ( $15.24 \%$ vs. $13.66 \%$, $\mathrm{p}=0.0001)$, drug abuse $(10.83 \%$ vs. $4.93 \%$, $\mathrm{p}<0.0001)$, alcohol abuse (10.83\% vs. $5.98 \%$, p $<0.0001)$, and current or past smoker $(43.87 \%$ vs. $27.45 \%$, p $<0.0001)$ than those without chronic periodontitis. Hospitalizations with chronic periodontitis were also associated with a higher percentage of CCI (Deyo's Charlson Co-morbidity) Index 2, 3, and $\geqslant 5$. US Hospitalizations in large, urban teaching hospitals and in the Midwest and South regions have a higher prevalence of chronic periodontitis compared to non-chronic periodontitis (Table 3).

Weighted frequency $(\%)$

Demographics of Patients

Age Mean \pm SD

$57.4 \pm 20.5$

Age Group (Years) (\%)

$18-34$

$35-49$

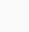

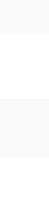

\section{Chronic Periodontitis}

$7020(0.01 \%)$
Non-chronic Periodontitis

$56492768(99.99 \%)$ $p$ values

$56499768(100)<0.0001$ 


\section{Cureus}

\begin{tabular}{|c|c|c|c|c|}
\hline $50-64$ & 36.18 & 23.91 & 23.91 & \\
\hline $65-79$ & 18.23 & 24.95 & 24.95 & \\
\hline$\geq 80$ & 6.70 & 16.74 & 16.74 & \\
\hline Gender (\%) & & & & $<0.0001$ \\
\hline Male & 59.19 & 41.06 & 41.07 & \\
\hline Female & 40.81 & 58.94 & 58.93 & \\
\hline Race (\%) & & & & $<0.0001$ \\
\hline White & 60.44 & 70.62 & 70.62 & \\
\hline African American & 25.93 & 15.21 & 15.21 & \\
\hline Hispanic & 11.21 & 10.96 & 10.96 & \\
\hline Asian or Pacific Islander & 1.90 & 2.60 & 2.60 & \\
\hline Native American & 0.51 & 0.61 & 0.61 & \\
\hline \multicolumn{5}{|l|}{ Characteristics of Patients } \\
\hline Median Household Income Category for patient's Zip code (\%) & & & & $<0.0001$ \\
\hline 0-25th percentile & 38.31 & 30.15 & 30.15 & \\
\hline 26-50th percentile & 26.40 & 26.75 & 26.75 & \\
\hline 51-75th percentile & 20.88 & 23.21 & 23.21 & \\
\hline 76-100th percentile & 14.41 & 19.88 & 19.88 & \\
\hline Primary Payer (\%) & & & & $<0.0001$ \\
\hline Medicare & 35.62 & 46.74 & 46.73 & \\
\hline Medicaid & 26.20 & 16.90 & 16.90 & \\
\hline Private Insurance & 21.27 & 27.68 & 27.68 & \\
\hline Other/Self-pay/No charge & 16.92 & 8.68 & 8.68 & \\
\hline Admission type (\%) & & & & $<0.0001$ \\
\hline Non- elective & 85.20 & 75.46 & 75.47 & \\
\hline Elective & 14.80 & 24.54 & 24.53 & \\
\hline Admission day (\%) & & & & 0.0981 \\
\hline Weekday & 78.92 & 79.71 & 79.71 & \\
\hline Weekend & 21.08 & 20.29 & 20.29 & \\
\hline \multicolumn{5}{|l|}{ Characteristics of Hospitals } \\
\hline Bedsize of hospital (\%) * & & & & $<0.0001$ \\
\hline Small & 12.11 & 16.22 & 16.22 & \\
\hline Medium & 21.01 & 28.05 & 28.05 & \\
\hline Large & 66.88 & 55.73 & 55.73 & \\
\hline Hospital Location \& Teaching Status (\%) & & & & 0.0001 \\
\hline Rural & 6.55 & 33.29 & 33.29 & \\
\hline Urban Non-teaching & 19.09 & 33.29 & 33.29 & \\
\hline Urban Teaching & 74.36 & 33.29 & 33.29 & \\
\hline Hospital Region (\%) & & & & $<0.0001$ \\
\hline Northeast & 18.09 & 20.10 & 20.10 & \\
\hline
\end{tabular}




\section{Cureus}

\begin{tabular}{|c|c|c|c|c|}
\hline Midwest & 23.15 & 20.49 & 20.49 & \\
\hline South & 42.31 & 40.13 & 40.13 & \\
\hline West & 16.45 & 19.27 & 19.27 & \\
\hline \multicolumn{5}{|l|}{ Comorbidities of Patients (\%) } \\
\hline Diabetes mellitus & 25.36 & 25.42 & 25.42 & 0.8978 \\
\hline Hypertension & 50.78 & 52.69 & 52.69 & 0.0014 \\
\hline Obesity & 15.24 & 13.66 & 13.66 & 0.0001 \\
\hline Hypercholesterolemia & 4.42 & 5.54 & 5.54 & $<0.0001$ \\
\hline Drug Abuse & 10.83 & 4.93 & 4.93 & $<0.0001$ \\
\hline Alcohol Abuse & 10.83 & 5.98 & 5.98 & $<0.0001$ \\
\hline Current or Past smoker & 43.87 & 27.45 & 27.45 & $<0.0001$ \\
\hline Deyo's Charlson Comorbidity Index (CCI) (\%) & & & & $<0.0001$ \\
\hline \multicolumn{5}{|l|}{0} \\
\hline 1 & 55.70 & 63.45 & 63.45 & \\
\hline 2 & 17.02 & 13.46 & 13.46 & \\
\hline 3 & 10.11 & 8.37 & 8.37 & \\
\hline 4 & 4.99 & 5.72 & 5.72 & \\
\hline$\geq 5$ & 12.81 & 9.00 & 9.00 & \\
\hline
\end{tabular}

\section{TABLE 3: Characteristics of chronic periodontitis in US hospitalizations (2013-2014)}

* Bedsize of hospital indicates the number of hospital beds which varies depending on hospital location (Rural/ Urban), teaching status (Teaching/ Non-teaching), and Region (Northeast/Midwest/ Southern/Western)

The numbers in the table are column percentage (\%) indicates a direct comparison between Chronic Periodontitis Vs. Non- Chronic Periodontitis amongst US hospitalizations between January 2013-December 2014.

\section{Outcomes of chronic periodontitis and cerebral atherosclerosis among US hospitalizations}

In univariate analysis, there was higher prevalence of cerebral atherosclerosis among chronic periodontitis compared to non-chronic periodontitis $(0.71 \%$ vs. $0.41 \%$, $\mathrm{p}<0.0001)$. Though the prevalence of acute ischemic stroke (AIS) and hemorrhagic stroke (HS) were higher in patients with non- chronic periodontitis (AIS: $2.21 \%$ vs. $1.97 \%, \mathrm{p}=0.1563$; HS: $0.57 \%$ vs. $0.46 \%, \mathrm{p}=0.1560$ ), the evidences of significance were weak (Table 4). Patients with cerebral atherosclerosis have higher frequency of AIS $(14.26 \%$ vs. $1.92 \%, \mathrm{p}<0.0001)$, TIA ( $3.55 \%$ vs. $0.68 \%, \mathrm{p}<0.0001)$ and HS $(1.48 \%$ vs. $0.45 \%, \mathrm{p}<0.0001)$ compared to no cerebral atherosclerosis (Table 5). 


\section{Cureus}

\begin{tabular}{|c|c|c|c|c|}
\hline & Chronic Periodontitis & Non-chronic Periodontitis & Total & p values \\
\hline Weighted trequency $(\%)$ & $7020(0.01 \%)$ & $56492 / 68(99.99 \%)$ & $56499768(100)$ & $<0.0001$ \\
\hline Cerebral Atherosclerosis & 0.71 & 0.41 & 0.41 & $<0.0001$ \\
\hline Acute Ischemic stroke (AIS) & 2.21 & 1.97 & 1.97 & 0.1563 \\
\hline Transient Ischemic stroke (TIA) & 0.57 & 0.69 & 0.69 & 0.2341 \\
\hline Hemorrhagic stroke (HS) & 0.57 & 0.46 & 0.46 & 0.156 \\
\hline
\end{tabular}

TABLE 4: Univariate Analysis of the Outcomes amongst Chronic Periodontitis.

The numbers in the table are column percentage (\%) indicates a direct comparison of outcomes (concurrent diagnoses) between Chronic Periodontitis Vs. Non- Chronic Periodontitis among US hospitalizations between January 2013-December 2014.

\begin{tabular}{|c|c|c|c|c|}
\hline & Cerebral Atherosclerosis & No Cerebral Atherosclerosis & Total & $p$ values \\
\hline Weighted frequency (\%) & $229765(0.41 \%)$ & $56270023(99.59 \%)$ & $56499788(100)$ & $<0.0001$ \\
\hline Acute Ischemic stroke (AIS) & 14.26 & 1.92 & 1.97 & $<0.0001$ \\
\hline Transient Ischemic stroke (TIA) & 3.55 & 0.68 & 0.69 & $<0.0001$ \\
\hline Hemorrhagic stroke (HS) & 1.48 & 0.45 & 0.46 & $<0.0001$ \\
\hline
\end{tabular}

\section{TABLE 5: Univariate Analysis of the Outcomes amongst Cerebral Atherosclerosis.}

The numbers in the table are column percentage (\%) indicates a direct comparison of outcomes (concurrent diagnoses) between Cerebral Atherosclerosis Vs. no- Cerebral Atherosclerosis among US hospitalizations between January 2013-December 2014.

\section{Regression model derivation}

The overall adjusted odds for cerebral atherosclerosis in chronic periodontitis patients among US hospitalizations were 2.48 (95\%CI:1.34-4.59, p<0.004), after adjusting for basic demographic with patientlevel variables, comorbidities, CCI, concurrent conditions compared to non- chronic periodontitis patients (Table 6).

\begin{tabular}{|c|c|c|c|c|}
\hline \multirow[t]{2}{*}{ Variables } & \multirow[t]{2}{*}{ Odds Ratio (OR) } & \multicolumn{2}{|c|}{ 95\% Confidence Interval (CI) } & \multirow[t]{2}{*}{$p$ values } \\
\hline & & LL & UL & \\
\hline No- Chronic Periodontitis & \multicolumn{4}{|l|}{ Reference } \\
\hline Chronic Periodontitis & 2.48 & 1.34 & 4.59 & 0.004 \\
\hline \multicolumn{5}{|l|}{ Age groups (years) } \\
\hline $18-34$ & \multicolumn{4}{|l|}{ Reference } \\
\hline $35-49$ & 3.01 & 2.38 & 3.81 & $<0.0001$ \\
\hline $65-79$ & 6.24 & 4.86 & 8 & $<0.0001$ \\
\hline$\geq 80$ & 5.77 & 4.43 & 7.53 & $<0.0001$ \\
\hline \multicolumn{5}{|l|}{ Gender } \\
\hline Female & \multicolumn{4}{|l|}{ Reference } \\
\hline Male & 1.08 & 1.05 & 1.1 & $<0.0001$ \\
\hline
\end{tabular}




\section{Cureus}

\begin{tabular}{|c|c|c|c|c|}
\hline \multicolumn{5}{|l|}{ Race } \\
\hline White & \multicolumn{4}{|l|}{ Reference } \\
\hline African American & 1.82 & 1.77 & 1.87 & $<0.0001$ \\
\hline Hispanic & 1.06 & 1.02 & 1.11 & 0.002 \\
\hline Asian or Pacific Islander & 1.16 & 1.09 & 1.24 & $<0.0001$ \\
\hline Native American & 0.9 & 0.77 & 1.06 & 0.216 \\
\hline \multicolumn{5}{|c|}{ Median Household Income Category for patient's Zip code } \\
\hline $0-25$ th percentile & \multicolumn{4}{|l|}{ Reference } \\
\hline 26-50th percentile & 0.98 & 0.96 & 1.01 & 0.167 \\
\hline 51-75th percentile & 0.99 & 0.96 & 1.02 & 0.446 \\
\hline 76-100th percentile & 1 & 0.97 & 1.03 & 0.888 \\
\hline \multicolumn{5}{|l|}{ Primary Payer } \\
\hline Medicare & \multicolumn{4}{|l|}{ Reference } \\
\hline Medicaid & 1.1 & 1.05 & 1.16 & 0 \\
\hline Private Insurance & 0.71 & 0.69 & 0.74 & $<0.0001$ \\
\hline Other/Self-pay/No charge & 0.89 & 0.84 & 0.95 & 0 \\
\hline \multicolumn{5}{|l|}{ Admission type } \\
\hline Non- elective & \multicolumn{4}{|l|}{ Reference } \\
\hline Elective & 0.53 & 0.51 & 0.54 & $<0.0001$ \\
\hline \multicolumn{5}{|l|}{ Admission day } \\
\hline Weekday & \multicolumn{4}{|l|}{ Reference } \\
\hline Weekend & 1.01 & 0.98 & 1.03 & 0.652 \\
\hline \multicolumn{5}{|l|}{ Bedsize of hospital } \\
\hline Small & \multicolumn{4}{|l|}{ Reference } \\
\hline Medium & 1.02 & 0.99 & 1.05 & 0.198 \\
\hline Large & 1.04 & 1.01 & 1.06 & 0.013 \\
\hline \multicolumn{5}{|l|}{ Hospital Location \& Teaching Status } \\
\hline Rural & \multicolumn{4}{|l|}{ Reference } \\
\hline Urban Non-teaching & 0.87 & 0.84 & 0.9 & $<0.0001$ \\
\hline Urban Teaching & 0.94 & 0.91 & 0.97 & $<0.0001$ \\
\hline \multicolumn{5}{|l|}{ Hospital Region } \\
\hline Northeast & \multicolumn{4}{|l|}{ Reference } \\
\hline Midwest & 1.22 & 1.18 & 1.25 & $<0.0001$ \\
\hline South & 1.21 & 1.17 & 1.24 & $<0.0001$ \\
\hline West & 1.05 & 1.02 & 1.09 & 0.005 \\
\hline \multicolumn{5}{|l|}{ Adjusted Comorbidities } \\
\hline Diabetes mellitus & 0.9 & 0.88 & 0.92 & $<0.0001$ \\
\hline AIDS & 0.11 & 0.09 & 0.15 & $<0.0001$ \\
\hline Renal failure (Acute/ Chronic/ ESRD) & 0.54 & 0.53 & 0.56 & $<0.0001$ \\
\hline Current or Past smoker & 0.68 & 0.66 & 0.69 & $<0.0001$ \\
\hline
\end{tabular}




\section{Cureus}

$\begin{array}{lllll}\text { Hypertension } & 1.49 & 1.45 & 1.53 & <0.0001 \\ \text { Obesity } & 0.63 & 0.6 & 0.65 & <0.0001 \\ \text { Hypercholesterolemia } & 1.04 & 1 & 1.08 & 0.03 \\ \text { Drug abuse/dependent } & 1.25 & 1.16 & 1.35 & <0.0001 \\ \text { Alcohol abuse/dependent } & 1.29 & 1.22 & 1.36 & <0.0001 \\ \text { CCI } & 1.38 & 1.38 & 1.38 & <0.0001 \\ \text { c- index } & 0.855 & & & \end{array}$

TABLE 6: Regression analysis showing association between chronic periodontitis with cerebral atherosclerosis

UL: Upper Limit; LL: Lower Limit; ESRD: End Stage Renal Disease; AIDS: Acquired Immunodeficiency Syndrome; CCI: Deyo's Charlson Comorbidity Index

The model is adjusted for basic demographic with patient-level variables, hospital-level variables such as hospital region, teaching status, and bed size, comorbidities, and $\mathrm{CCl}$.

Table 7 exhibits a multivariate regression analysis showing the association of chronic periodontitis and cerebral atherosclerosis with cerebrovascular diseases (AIS, TIA, and HS). Cerebral atherosclerosis patients were associated with higher odds of TIA attack (aOR: 2.40; 95\%CI: 2.28-2.53; p<0.0001), AIS (aOR: 3.35; 95\%CI: $3.25-3.45$; $\mathrm{p}<0.0001$ ), and HS (aOR: 1.51 ; 95\%CI: $1.39-1.64$, $\mathrm{p}<0.0001$ ) compared to patients without cerebral atherosclerosis, though there was no direct evidence of relationship between chronic periodontitis and cerebrovascular disorders (TIA- aOR: 0.99, $\mathrm{p}=0.980$; AIS-aOR: 1.02 ; $\mathrm{p}=0.919$; HS- aOR: $1.02, \mathrm{p}=0.956$ for Model 1, 2, and 3, respectively).

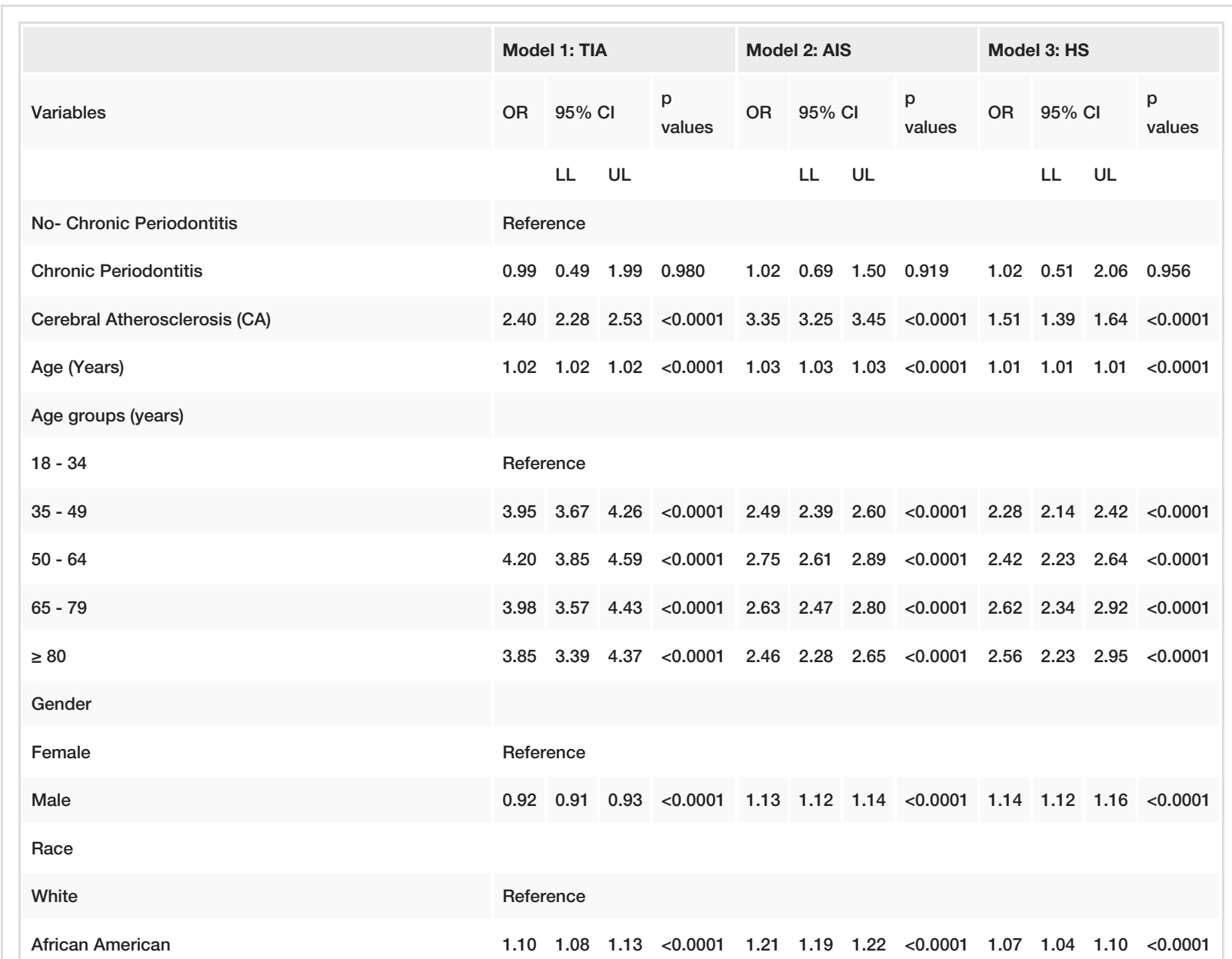




\section{Cureus}

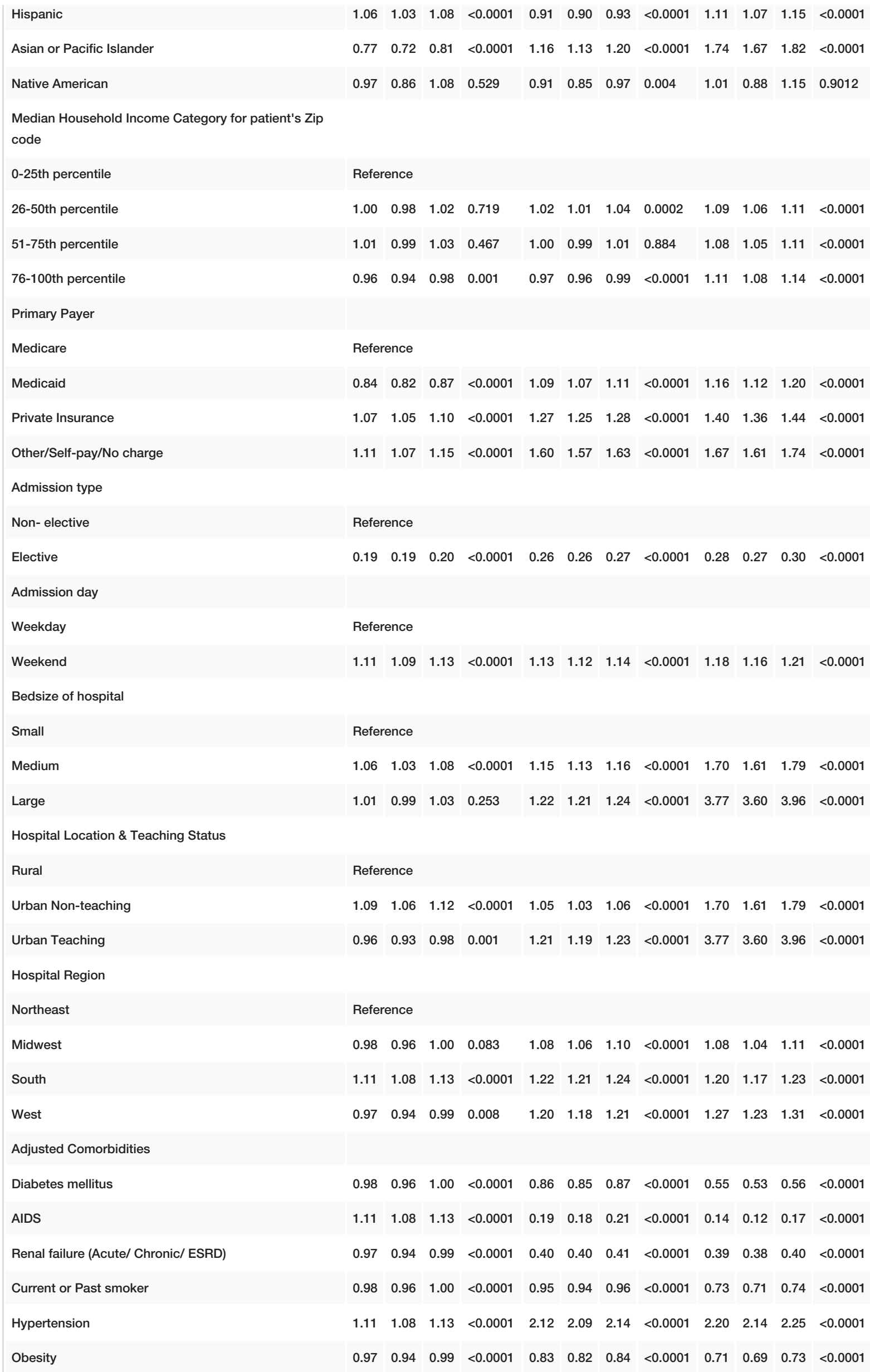




\section{Cureus}

\begin{tabular}{|c|c|c|c|c|c|c|c|c|c|c|c|c|}
\hline Hypercholesterolemia & 0.98 & 0.96 & 1.00 & $<0.0001$ & 1.21 & 1.19 & 1.23 & $<0.0001$ & 0.90 & 0.86 & 0.93 & 0.030 \\
\hline Drug abuse/dependent & 1.11 & 1.08 & 1.13 & $<0.0001$ & 1.06 & 1.03 & 1.09 & $<0.0001$ & 1.24 & 1.19 & 1.30 & $<0.0001$ \\
\hline Alcohol abuse/dependent & 0.97 & 0.94 & 0.99 & $<0.0001$ & 0.85 & 0.83 & 0.87 & $<0.0001$ & 0.98 & 0.94 & 1.02 & 0.2436 \\
\hline $\mathrm{CCl}$ & 1.17 & 1.17 & 1.18 & $<0.0001$ & 1.31 & 1.31 & 1.31 & $<0.0001$ & 1.32 & 1.32 & 1.32 & $<0.0001$ \\
\hline c- index & 0.772 & & & & 0.803 & & & & 0.798 & & & \\
\hline
\end{tabular}

TABLE 7: Regression analysis showing relationship of chronic periodontitis and cerebral atherosclerosis with cerebrovascular diseases

TIA: Transient Ischemic Attack; AIS: Acute Ischemic Stroke; HS: Hemorrhagic Stroke

OR: Odds Ratio; CI: 95\% Confidence Interval; UL: Upper Limit; LL: Lower Limit; ESRD: End-Stage Renal Disease; AIDS: Acquired Immunodeficiency Syndrome; CCl: Deyo's Charlson Comorbidity Index

The model is adjusted for basic demographic with patient-level variables, hospital-level variables such as hospital region, teaching status, and bed size, comorbidities, and $\mathrm{CCl}$.

\section{Accuracy of the model}

c-statistic was used to validate the accuracy of the regressions. It was $0.772,0.803,0.798$ for adjusted model 1,2 and 3 respectively. All models have c-index $>0.7$, which indicates a good model fit.

\section{Discussion}

With increasing prevalence, chronic periodontitis is arising as a major public health concern contributing to the global burden of chronic diseases. According to the CDC, nearly half of the Americans aged 30 or older have periodontitis [3]. Chronic inflammation due to periodontal disease has a causal relation to atherosclerosis mediated by changes in inflammation and the immune response, with subsequent causative relationships to stroke and heart disease [17,18]. Prior observational studies have shown that poor periodontal health status is associated with increased stroke risk. In our large population-based crosssectional study, we aimed to investigate the link between chronic periodontitis and cerebrovascular disease. Further assessment of risk profiles for periodontal disease in adults in the United States shows male sex, current cigarette smoking, and diabetes mellitus as important risk factors for periodontal disease [19].

Chronic periodontitis represents a systemic burden of bacteria, endotoxin, and other bacterial products, which can induce an abundant production of proinflammatory cytokines leading to inflammatory cell proliferation into large arteries and stimulate the hepatic synthesis of clotting factors, thus contributes to atherogenesis and thromboembolic events $[1,20,21]$. Chronic periodontal disease patients have increased levels of inflammatory cytokines like TNF - alpha, IL- 1, and IL- 6 which leads to fibrin deposition and atherogenic trigger response $[1,22]$. These hypotheses of pathogenesis help to establish the association of the periodontal disease with cardiovascular disease, cerebrovascular disease, and atherosclerosis. This may explain our study results of the increased incidence of atherosclerosis in chronic periodontitis.

In this large population-based cross-sectional study we found a direct association between chronic periodontitis with atherosclerosis and in-direct relation to a higher incidence of cerebrovascular disease. US hospitalizations with chronic periodontitis had a higher prevalence in the age group 50-64 years, male, African American, and 0-25th percentile median household income category patients compared to nonchronic periodontitis. Various studies have data on the higher prevalence of chronic periodontitis in African Americans due to poor dental hygiene [23]. In our study, chronic periodontitis was also associated with other comorbidities such as diabetes, hypertension, hyperlipidemia, obesity, drug abuse, alcohol abuse, and tobacco smoking. Interestingly, we found a 2.48 times more risk of cerebral atherosclerosis among chronic periodontitis as compared to no chronic periodontitis. Higher prevalence of AIS, TIA, and HS were noted in chronic periodontitis, but the evidence was not significant $(\mathrm{p}=0.1560)$. Higher prevalence of AIS, TIA, and HS are seen in patients with cerebral atherosclerosis as compared to patients without atherosclerosis, with odds of 3.35, 2.40, 1.51 in AIS, TIA, and HS respectively. In our study, we could not establish a direct relationship between cerebrovascular diseases and chronic periodontitis. Therefore, chronic periodontitis does not directly increase the risk of cerebrovascular diseases but increases the risk of cerebral atherosclerosis which is associated with increased risk of cerebrovascular diseases.

Several observational meta-analysis studies have shown that chronic periodontitis and poor periodontal health status is associated with an increased risk for stroke and cardiovascular diseases [24,25]. A large population-based, nationwide study in Taiwan identified the periodontal disease as an important risk factor for ischemic stroke incidence and showed that periodontal treatment lowered the risk of stroke, significantly among young adults [26]. Grau et al. carried out a case-control study in the German population, in which 
increasing severity of periodontitis was associated with an increased risk of ischemic stroke after adjustment for age, gender, number of teeth, and other covariables [27]. A more recent meta-analysis of two cohort studies found that periodontal disease increased the risk of ischemic strokes by 1.6-fold [5].

It is possible that an increased risk of thrombotic stroke may be secondary to atherothrombosis in the cervicocerebral vasculature and cardioembolic stroke association may be because of coronary artery disease or atrial fibrillation related to periodontal disease-induced inflammation. A recent study by Sen et al. cohort of the ARIC database was studied over a period of 15 years which demonstrated an increased hazard of cardioembolic and thrombotic stroke in chronic periodontitis. The study also shows regular dental care use continued to be associated with lower rates of ischemic stroke [28].

Our study analysis showed no direct linkage between cerebrovascular diseases and chronic periodontitis, but chronic periodontitis was significantly associated with an increased prevalence of cerebrovascular diseases. This contradictory finding may have arisen as periodontitis is usually managed on an outpatient basis, so inpatient data of chronic periodontitis prevalence might be underrepresented. There is a need to be more vigilant in diagnosing chronic periodontitis on both inpatient and outpatient basis. Early detection of chronic periodontitis, treatment, and stressing the need for dental hygiene can be emphasized which ultimately will lead to a reduction in the risk of cerebrovascular events.

We strongly recommend that chronic periodontitis should be identified as an important risk factor for cerebral atherosclerosis and a possible risk for cerebrovascular diseases (AIS, TIA, and HS). Poor oral hygiene which contributes to periodontal disease is a modifiable risk factor. Evidence from randomized controlled trials has established that intensive periodontal treatment improves systemic inflammation, high blood pressure, lipid profile, and endothelial dysfunction [29,30], further reducing the risk of atherosclerotic disease. Frequent tooth brushing and regular dental care will potentially reduce the risk of cerebrovascular diseases.

\section{Strength and Limitations}

To the best of our knowledge, this would be the first US-based large population cross-sectional study to report the relationship between chronic periodontitis and cerebral atherosclerosis. NIS Data represent the largest inpatient database. This study does have some limitations. We were not able to establish a direct causal relationship between chronic periodontitis and cerebrovascular disease with good statistical power. But this study successfully puts forward an indirect relationship between chronic periodontitis and cerebrovascular diseases. Diagnosis of chronic periodontitis in the NIS database is the physiciandocumented diagnosis, hence susceptible to coding errors, and with chronic periodontitis being usually managed in an outpatient setting, might have been underdiagnosed as a hospital problem. We were not able to quantify the severity of chronic periodontitis.

\section{Conclusions}

Our study found a significant association of cerebral atherosclerosis with chronic periodontitis.

Furthermore, we could not establish the directly associated risk of chronic periodontitis with cerebrovascular disease, but it increases the burden of cerebrovascular diseases through evidently increasing the risk of cerebral atherosclerosis. Early identification of chronic periodontitis and atherosclerotic risk factors helps to mitigate the risk of cerebrovascular diseases.

\section{Additional Information \\ Disclosures}

Human subjects: All authors have confirmed that this study did not involve human participants or tissue. Animal subjects: All authors have confirmed that this study did not involve animal subjects or tissue. Conflicts of interest: In compliance with the ICMJE uniform disclosure form, all authors declare the following: Payment/services info: All authors have declared that no financial support was received from any organization for the submitted work. Financial relationships: All authors have declared that they have no financial relationships at present or within the previous three years with any organizations that might have an interest in the submitted work. Other relationships: All authors have declared that there are no other relationships or activities that could appear to have influenced the submitted work.

\section{References}

1. Cardoso EM, Reis C, Manzanares-Cespedes MC: Chronic periodontitis, inflammatory cytokines, and interrelationship with other chronic diseases. Postgrad Med. 2018, 130:98-104. 10.1080/00325481.2018.1396876

2. Pihlstrom BL, Michalowicz BS, Johnson NW: Periodontal diseases. Lancet. 2005, 366:1809-1820. 10.1016/S0140-6736(05)67728-8

3. Eke PI, Dye BA, Wei L, Thornton-Evans GO, Genco RJ: Prevalence of periodontitis in adults in the United States: 2009 and 2010. J Dent Res. 2012, 91:914-920. 10.1177/0022034512457373

4. Leng WD, Zeng XT, Kwong JS, Hua XP: Periodontal disease and risk of coronary heart disease: An updated 
meta-analysis of prospective cohort studies. Int J Cardiol. 2015, 201:469-472. 10.1016/j.ijcard.2015.07.087

5. Lafon A, Pereira B, Dufour T, Rigouby V, Giroud M, Bejot Y, Tubert-Jeannin S: Periodontal disease and stroke: a meta-analysis of cohort studies. Eur J Neurol. 2014, 21:1166-1157. 10.1111/ene.12415

6. Bui FQ, Almeida-da-Silva CLC, Huynh B, et al.: Association between periodontal pathogens and systemic disease. Biomed J. 2019, 42:27-35. 10.1016/j.bj.2018.12.001

7. Kelly JT, Avila-Ortiz G, Allareddy V, Johnson GK, Elangovan S: The association between periodontitis and coronary heart disease: a quality assessment of systematic reviews. J Am Dent Assoc. 2013, 144:371-379. 10.14219/jada.archive.2013.0130

8. Leivadaros E, van der Velden U, Bizzarro S, et al.: A pilot study into measurements of markers of atherosclerosis in periodontitis. J Periodontol. 2005, 76:121-128. 10.1902/jop.2005.76.1.121

9. Mattila KJ, Nieminen MS, Valtonen VV, et al.: Association between dental health and acute myocardial infarction. BMJ. 1989, 298:779-781. 10.1136/bmj.298.6676.779

10. Kelly BM, Pangilinan PH, Jr., Rodriguez GM: The stroke rehabilitation paradigm. Phys Med Rehabil Clin N Am. 2007, 18:631-650. 10.1016/j.pmr.2007.07.006

11. Sfyroeras GS, Roussas N, Saleptsis VG, Argyriou C, Giannoukas AD: Association between periodontal disease and stroke. J Vasc Surg. 2012, 55:1178-1184. 10.1016/j.jvs.2011.10.008

12. Janket SJ, Baird AE, Chuang SK, Jones JA: Meta-analysis of periodontal disease and risk of coronary heart disease and stroke. Oral Surg Oral Med Oral Pathol Oral Radiol Endod. 2003, 95:559-569. 10.1067/moe.2003.107

13. Khader YS, Albashaireh ZS, Alomari MA: Periodontal diseases and the risk of coronary heart and cerebrovascular diseases: a meta-analysis. J Periodontol. 2004, 75:1046-1053. 10.1902/jop.2004.75.8.1046

14. Leira Y, Seoane J, Blanco M, Rodriguez-Yanez M, Takkouche B, Blanco J, Castillo J: Association between periodontitis and ischemic stroke: a systematic review and meta-analysis. Eur J Epidemiol. 2017, 32:43-53. 10.1007/s10654-016-0170-6

15. Patel U, Kodumuri N, Malik P, et al.: Hypocalcemia and Vitamin D deficiency amongst migraine patients: a nationwide retrospective study. Medicina. 2019, 55:407. 10.3390/medicina55080407

16. Birman-Deych E, Waterman AD, Yan Y, Nilasena DS, Radford MJ, Gage BF: Accuracy of ICD-9-CM codes for identifying cardiovascular and stroke risk factors. Medical care. 2005, 43:480-485. 10.1097/01.mlr.0000160417.39497.a9

17. Mattila KJ, Pussinen PJ, Paju S: Dental infections and cardiovascular diseases: a review . J Periodontol. 2005, 76:2085-2088. 10.1902/jop.2005.76.11-S.2085

18. Loos BG: Systemic markers of inflammation in periodontitis . J Periodontol. 2005, 76:2106-2115. 10.1902/jop.2005.76.11-S.2106

19. Eke PI, Dye BA, Wei L, et al.: Update on prevalence of periodontitis in adults in the United States NHANES 2009 to 2012. J Periodontol. 2015, 86:611-622. 10.1902/jop.2015.140520

20. Mustapha IZ, Debrey S, Oladubu M, Ugarte R: Markers of systemic bacterial exposure in periodontal disease and cardiovascular disease risk: a systematic review and meta-analysis. J Periodontol. 2007, 78:2289-2302. 10.1902/jop.2007.070140

21. Herzberg MC, Meyer MW: Effects of oral flora on platelets: possible consequences in cardiovascular disease . J Periodontol. 1996, 67:1138-1142. 10.1902/jop.1996.67.10s.1138

22. Tonetti MS, Van Dyke TE: Periodontitis and atherosclerotic cardiovascular disease: consensus report of the Joint EFP/AAP Workshop on Periodontitis and Systemic Diseases. J Periodontol. 2013, 84:24-29. 10.1902/jop.2013.1340019

23. Nalliah RP, Virun V, Dhaliwal G, Kaur H, Kote A: Fifteen-year gap between oral health of blacks and whites in the USA. J Invest Clin Dent. 2019, 10:e12415. 10.1111/jicd.12415

24. You Z, Cushman M, Jenny NS, Howard G, Regards: Tooth loss, systemic inflammation, and prevalent stroke among participants in the reasons for geographic and racial difference in stroke (REGARDS) study. Atherosclerosis. 2009, 203:615-619.10.1016/j.atherosclerosis.2008.07.037

25. Wu T, Trevisan M, Genco RJ, Dorn JP, Falkner KL, Sempos CT: Periodontal disease and risk of cerebrovascular disease: the first national health and nutrition examination survey and its follow-up study. Arch Intern Med. 2000, 160:2749-2755. 10.1001/archinte.160.18.2749

26. Lee YL, Hu HY, Huang N, Hwang DK, Chou P, Chu D: Dental prophylaxis and periodontal treatment are protective factors to ischemic stroke. Stroke. 2013, 44:1026-1030. 10.1161/strokeaha.111.000076

27. Grau AJ, Becher H, Ziegler CM, et al.: Periodontal disease as a risk factor for ischemic stroke . Stroke. 2004, 35:496-501. 10.1161/01.Str.0000110789.20526.9d

28. Sen S, Giamberardino LD, Moss K, et al.: Periodontal disease, regular dental care use, and incident ischemic stroke. Stroke. 2018, 49:355-362. 10.1161/strokeaha.117.018990

29. D'Aiuto F, Parkar M, Nibali L, Suvan J, Lessem J, Tonetti MS: Periodontal infections cause changes in traditional and novel cardiovascular risk factors: results from a randomized controlled clinical trial. Am Heart J. 2006, 151:977-984. 10.1016/j.ahj.2005.06.018

30. Tonetti MS, D'Aiuto F, Nibali L, et al.: Treatment of periodontitis and endothelial function . N Engl J Med. 2007, 356:911-920. 10.1056/NEJMoa063186 